\title{
Integrative Information Retrieval with a Consumer Strategy Approach
}

\author{
Sridevi $\mathrm{K} \mathrm{N}^{1,2, *}$ Prakasha $\mathrm{S}^{3}$ \\ ${ }^{1}$ CSE Department $R \&$ D Center, RNSIT, Bengaluru, India \\ ${ }^{2}$ Department of ISE, VEMANA Institute of Technology, Bengaluru, India \\ ${ }^{3}$ Department of ISE, RNSIT, Bengaluru, India \\ *Corresponding author. Email: sridevi.kn23@gmail.com
}

\begin{abstract}
In this study, Information Retrieval has become an integral part of our life. We require these technologies to make our digital life comfortable in this fast running technology era. We have tried to describe a consumer methodology to coordinating information retrieval, wherein the emphasis is placed on the consumer's behaviours rather than the subsystem. This process explains the novel methodologies in the field of Information Retrieval, this methodology has been more efficient over the old ways which seems to lack in few aspects. This strategy entails using transcription and effective interactions to facilitate information exchange between consumers mostly during information retrieval process. We built an ecosystem for consumers to reciprocate their understanding both sequentially and interdependently during in the description and validation of information asymmetry, the selection of an information technology being used for research, the composition and reinterpretation of quests to communicate their responses suggest related to information system selected; and the formulation and reinterpretation of quests to convey their signs indicate based on the data which is chosen. and during the evaluation of information relevancy to the resolution of their information problem. Here have presented a novel approach in detail in multiple sections ahead in this paper.
\end{abstract}

Keywords: Information Retrieval, Collaborative Information Behavior, Collaborative Information Retrieval, Knowledge Sharing, Interpersonal Communication, Collective Intelligence.

\section{INTRODUCTION}

Information Retrieval (IR) is a notion that can be found in a variety of disciplines, agencies, industries, and other settings. It is impossible to overstate the relevance of knowledge discovery in any group. Every problem definition in any developmental organization can be transformed into an information problem, i.e., whose data is obtained to make the best choice possible on a perceived task. Information retrieval is the result of an issue with information.

Increased advances in digital technology and approaches have resulted in some progression in IR studies, as well as a slew of new research questions. One such issue is the misalignment regarding real-world IR practice and the conception and growth of information retrieval systems (IRS). This disparity can be seen in the way users are modelled in IRS and in how users' IR behavior is understood. In a conventional IRS, all customers are given a system approach that is not representative of reality. Individual users were modelled in customized IRS [1], allowing the ability to function to users' questions tailored to individual. Content - based filtering and advisory mechanisms, in which a user gets a suggestion determine the similarity of his information seeking operations to the masses grouping of historically crowd behavior, have been improved further. This global community method seeks to assist a member in solving his dilemma by recommending solutions that other consumers with different challenges have used in the background.

Researchers found that people in real-world environments exhibit collaborative information behavior based on the observations of their information behavior (CIB). Given the enormous data systems and strong internet sites websites on the net, users are still caught in a loop of interviewing information sources and interviewing workers to help them solve their information problem. When a user has a signs indicate in an organization with three or more individuals, for 
example, he usually begins by asking his coworkers if they know how to handle the problem.

The extent to which he discusses the nitty-gritty of his problem is determined by his level of confidence in his coworkers and the sensitivity of the information problem. When he describes his predicament to his coworkers, they try to get a better understanding of it by providing more information. Whether they have adequate experience to assist him, they offer possible solutions to his predicament or information sources he may consult [2]. He gets caught in a cycle of asking data sources and people to help him solve his knowledge dilemma. Here as seen on the illustration that attempting to resolve a finding suggest necessitates a thorough view of the issues, i.e. the users regarding requirement. One of its biggest problems in IR would that be.

The majority of IR systems will not address the question of assisting people in better understanding their problems before initiating queries [3-5]. Despite the fact that some define requirements query combining and query recommendation [6-8], all of them are still based on the user's first query. If the first query is a blatant misunderstanding of the solution, query recommendations and merge is now nothing more than a shoddy platform on which to construct. The second concern is that customers don't really rely on a specific IRS to solve their problems in real life. They seek advice from as many IRS offices as necessary [9-11].

In section 2 of this document, a certain aspect is identifying current research. The notion of CIR is explained in Section 3. Chapter 4 will concentrate on CIR users, and Chapter 5 will clarify all the cooperative information retrieval system (CIRS), and Section 6 will wrap things up.

\section{RELATED WORKS}

There have been several efforts to facilitate cooperation in the field of IR. Most of these efforts, as described in the title, are based on mutual intelligence, in which users' operations are recorded in intended to facilitate other user who might have been experiencing a similar experience. The issue of interpretation of users' information is going expressed as queries is still unanswered.

Romano et al. [12] performed out that one of the first studies on CIR observing of person conducting inquiries demonstrates that independently of it when and that a searches arises, either in a bookstore, in business organizations, or in visitors wandering around neighborhoods, reference materials are also used jointly to seek actionable insights," they write [10]. A Cooperation Information Retrieval Environment was created by them (CIRE)that helps organizations find and retrieve the data they need to collaborate on projects. They studied users' experience with IRS and Group
Support Systems (GSS) and concluded that the advantages of these different systems could be merged in a CIR environment. As a result, the CIRE prototypes was created, which combines the paradigms of these three zones. There are certain constraints to the procedure. The most significant restriction is that it only allows for asynchronous collaborating. The drawback is that it is intended for small work groups, and users' inquiries are conducted using the Altavista search engine. Users are unable to choose their preferred search engine.

In their own case, David and Bueno [12-14] recommended a CIR system (METIORE) based on the idea of computer interaction communications, in which IR upgrades are no longer solely the responsibility of the system, but also of the users. METIORE is a bibliographic information retrieval solution that performs collaboratively. Users should work together to extract data from a reference list using this system. METIORE necessitates the use of a special database schema, such as XML or a trust database. It doesn't have a text messaging search function.

Twidale, Nichols, and Paice [12] created ARIADNE (Annotatable Retrieval of Information and Database Navigation Environment), a mechanism for cooperative database searching teach the students. It's an application software for visualizing the process of finding data. The model was installed to be used for database searching, although most information retrieval activities are now carried out via the internet through search results as well as other online IRS.

Ginsburg [15] created the Annotate system, that allows users to annotate documents and track their worldwide usage history. The authors asserted the difficulties of internet web pages in avoiding the democratic rule of the Web's Hypertext Transfer Protocol (HTTP), that making it impossible to keep track of users' searching session and, as a result, does not allow users to share their thoughts. The annotate method is designed by the author as an ability to increase a collaborative dimension to internet full text searches.

Amershi and Morris [1] created CoSearch, a device that includes easily accessible technologies like smart phones and additional mice to enhance the experience of professional and non-cooperative search engine. Robert [15-18] looked at how homogenous technologies would collaborate on annotation creating and use it for judgement and exchanging interactions.

Other studies have been carried out to clarify the basic problems in CIR. Karamuftouglu [19] claims that "The supply and demand of knowledge are said to be the fundamental intellectual challenges of IR. Knowledge production is essentially a cooperative process that is firmly ingrained in the practices of a group of people who make up a domain. Fidel et al. [20] conducted a thorough investigation of CIR in natural environments. They 
guided their inquiry with a work-centered theoretical framework method called work assessment, but theirs research was centered on a working environment, which was believed does not highlight the fact that specific users manifest $\mathrm{CIB}$ in their quest to resolve their information problem.

Cooperation labeling, a method in which users add information in the form of phrases to social network, is yet another strategy that is gaining some traction in contemporary web 2.0 and network topology. Observable behaviors in internet usage, tag rhythms, types of tags used, bursts of prominence in timestamping, and exceptional consistency in the geographical distribution of labels within the same given urI were found by Golder and Huberman [16-17] in their understanding of the structure of collective trigging mechanisms using del.icio.us as a test case.

In [26] the concept of information retrieval has been applied in a very innovative way where collaborated the inference drawn from the principles in which it can help in the research study. Here the author tried to evaluate the feasibility of the performance and other traditional old retrieval techniques which gives us a profound knowledge in our innovative field. Here they have used it for vehicular movement and certain navigation techniques which helps them along with IOT technology.

In [20] as seen can get some insights which can help in the research, here they have discussed Information retrieval methods on a unique CLAD method on text analogs using the test document and indexed documents, here can be learnt based on the patterns of how can get some working on information retrieval methods in real time texting, this helps in research where it can be incorporated the best possible customer strategy approaches.

In [24] the authors have given a profound explanation to the analysis of information retrieval system has produced an optimized way to obtain an efficient search based on various documents. There are certain mathematical calculations which are provided by the authors in finding the efficient way to get the desired document based on IR approach, these insights help us to understand the approach in a better way and gives us a path to build the analysis.

\section{INFORMATION RETRIEVAL METHODOLOGY}

To comprehend CIR and determine if the above systems are truly CIR systems, the two underlying principles of improved information retrieval must be defined. Information retrieval [21] can indeed be way which understood in a larger context, according to Fidel et al. [4], to also include procedures such as critical thinking, interpretation of information is going, query formulation, retrieve collaborations, assessment, presentation of the results, and implementing outcomes to address a sign indicate. Cooperation, on the other hand, can be understood as the act of collaborating together toward a common goal. It's crucial to understand the distinction between collaboration and cooperation.

People in partnership talk about their experiences more towards a common goal, whereas customers in partnership does not seem to have a specific purpose [11]. They must share their information, but both may not even have a common goal. According to these meanings, the majority of the systems mentioned are indeed cooperative systems in which users share their expertise but do not have a common goal. Unlike other techniques to CIR, as expected customers to begin with an integrated information dilemma and a common insight into the problem while moving onto that functional strategies, recommendation, and combining. This is why the starting point in system is the problem definition interface described in part 5.

CIR, in this opinion, consists of tools and procedures for regulating users' social structures in the information retrieval process in order to encourage directly collaboration and communication amongst consumers. The focus isn't just on "collective actions," [24] as well as on direct cooperation between users, which relates to the disclosure of tacit and explicit knowledge.

Information, teamwork, cooperation modes, and cooperative organizational learning were listed as 4 main factors to be taken in CIR in prior projects [14]. As addressed the different types of knowledge that can be exchanged throughout cooperative knowledge discovery in another paper [13]. Skill level, device knowledge, query technique understanding, relevant system knowledge, and knowledge of possible partners are all examples of this awareness.

- The degree to which a consumer comprehends a search subject is referred to as knowledge base. This refers to a property's understanding of reality, definitions, and terms. High domain awareness helps a consumer to even more efficiently analyze collected outcomes and offers a more complex range of concepts and terminology for query formulation [17] [12].

- Awareness of search techniques represents a user's ability to plan his or her search. This involves research methodology, device choice, agent use, apostrophes, datatype signs, and relevant search adjustment and extension [19].

- A participant's awareness of the IRS's features and functionality (e.g. new word, word collection, versatile search database, Boolean database support, display result overview, etc.) and also how the process will allow him/her achieve his/her searching goals is expressed in system knowledge. 
- Information resource knowledge reflects a user's knowledge of locating outline databases or web resources or search system that can be useful for resolving an information problem [21-28].

- Increasing IRS consumer possess differing levels of the kinds of data described earlier when it comes to getting potential collaborators. The first challenge for a user's who wish to collaborate with some other user is deciding who he should coordinate with, i.e. a possible collaborator. Determining a possible associate is a characteristic of how well a user can mine the backgrounds and prototypes of several other consumers to assess their extent of the abovementioned types of information [29-30].

\section{MODELLING CONSUMERS IN CIR TECHNIQUES}

"Faith" is amongst the most significant elements in teamwork. A consumer must have a certain degree of confidence in order to communicate his knowledge issue to others, perhaps because of the individual's competence or and there is no risk in discussing with someone. I might regard somebody as capable of assisting me in solving my problem, but since I always regard him as a possible opponent, I may be reluctant to discuss my dilemma with him rather than attempt to hide my major issue or purpose. Collaboration has both a social and psychological component [31-34].

This is an issue that can only be immediately fixed even though it is linked to the values, so this can be helped to people obtain a reasonable degree of trust in other applications even by ways they are modelled, which reflects the information the process has on them. This is the first "Trust level" to start the collaboration process. Building trust is an ongoing skill that evolves over process as a function of users' interactions communicating with one another [35].

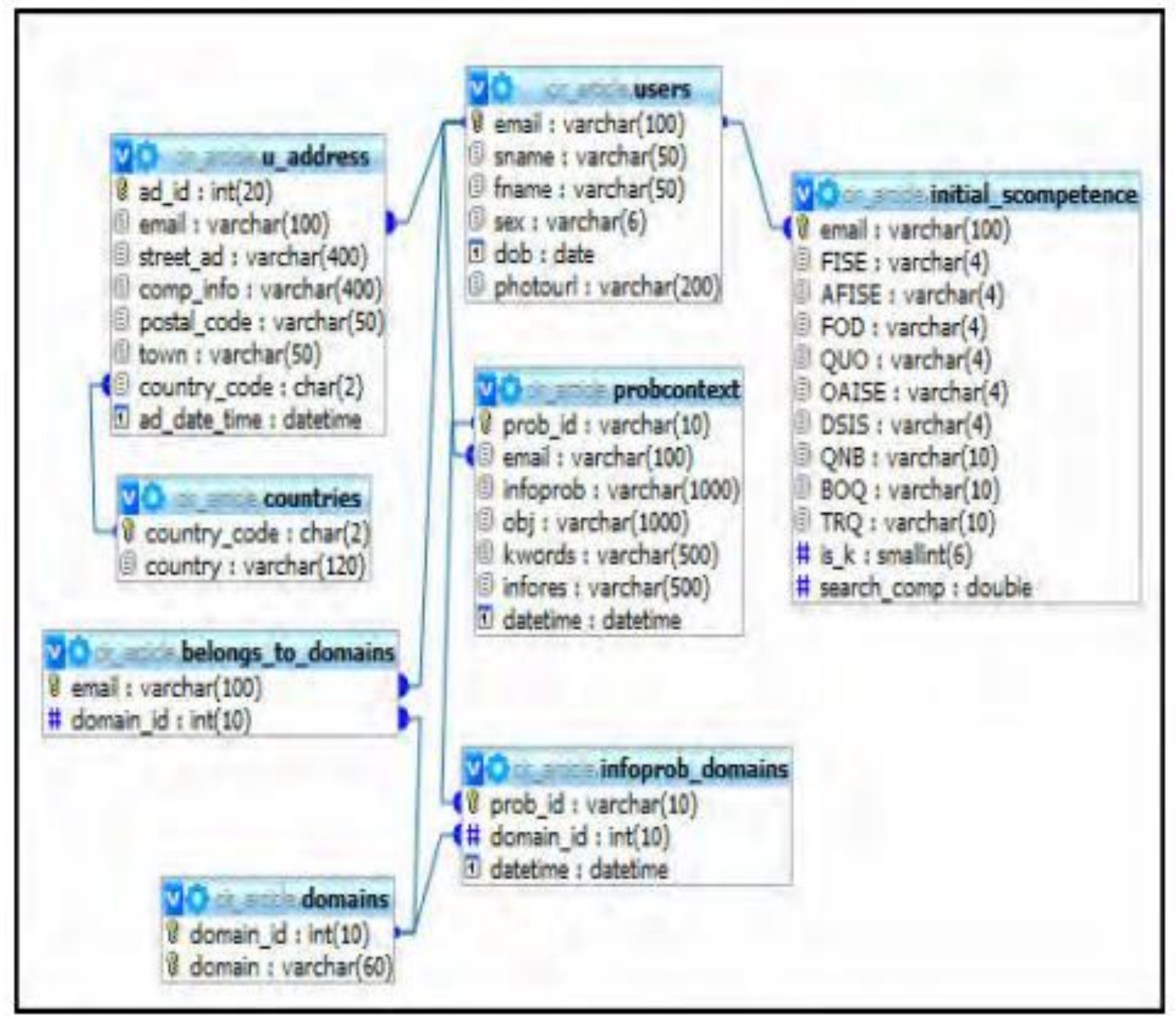

Figure 1 Relational Graph depicting attribute-value pair information in knowledge base 
A customer's personally identifiable information, including such name, first names, gender, birthdate, contact, and photograph, is stored in their database. The customer's geographic background is split into 2 parts: the destination just at time of enrollment and the position at every sign-in. A user interface is a physical reminder of all the people for whom he works on the device. This is a criterion for determining a user's authority and expertise in relation to an application domain. It also aids in the growth of a user's trust. A user who is a part of a domain's guru network may be intuitively considered to be considerably knowledgeable in the domain [36-38]. System knowledge, information source knowledge, domain knowledge, search competence and knowledge of collaborators have been explained above.

When a consumer is logging on the framework, as chosen a series of questionnaires as well as some side principles to assign marks to both the users. based on their initial step query experiences and skills of data sources.

Consider the following scenario:

"Are we aware of the significance of quotes in functional strategies on an online search?"

Question 1: "Are we aware of the relevance of speech marks in functional strategies on an online search?"

"Will this query extend or restrict the answer sequence number?" is the second question.

"Sarah mostly in swamp" is a question.

If the consumer protection "correct" to request 1 and "deepen" to request 2, it means he or she does not recognize the necessity of quote marks in functional strategies on an online search. However, if the user answers "sure" to request 1 and "close" to request 2, the algorithm awards him 2 points as his initial step authoritative in search skill [39]. Other questions were asked to see whether the user could define Boolean queries and truncation queries, as well as formulate them. The attribute-value pair is used to reflect user information in the knowledge base. Figure 1 depicts a relational graph of this influence.

There have been two features to this service's portrayal of consumers [40-41]. First is the entry level (information that could be gather from either the user when registered upon this network), and the other is information gleaned from the customer's behaviors mostly on structure. His problem statement, questions, descriptions, and connections with many other consumers are all excellent. Here this may decide how competent a consumer is in locating good sources in a specific domain related to the problem framework description and the suggested sources of information even by consumer [42].

\section{METHODOLOGY AND DISCUSSION}

\subsection{Collaboration Paradigms}

The user centered CIRS allows user to collaborate in three modes:

- Open group

- Closed group

- Peer-to-peer

When a user first logs in to the framework, he is immediately assigned to the open party. By design, all members are members of the open community. This is a place where users can read about what's being done and who else belongs to the very same category as themselves. A consumer in the inclusive society may request cooperation from any other device user. It is not a given that any proposal for direct cooperation would be met with enthusiasm. What we're attempting to depict here is a normal situation in which a user inputs an atmosphere and simply asks for assistance in identifying details. "How do I get to Brown Main road?" for instance.

Closed group: A closed group may be either a preset team working on a mutual pre - specified question or a system user - generated who found a shared attraction through their experiences in the established party and can share their skills at any creatively to answer their users' signs indicate. Any contact in this phase is visible to all members of the community.

Peer-to-peer: This method is designed to accommodate openness in knowledge communications and information sovereignty security. In this model, a client collaborates with some other client on a peer-topeer basis to solve his knowledge problem. The relations behind them are also accessible to us, and the information created during this interaction is not stored in a global fashion.

\subsection{Information Problem Context Definition}

Here platform's upper section is represented in Figure 2. A person's first step is to identify his or her knowledge issue. This is how it was modelled:

$\begin{array}{ll}\text { 1. } & \text { Information problem } \\ \text { 2. } & \text { Objective } \\ \text { 3. } & \text { Date and time } \\ \text { 4. } & \text { Information systems to use for search } \\ \text { 5. } & \text { Intended keywords for search } \\ \text { 6. } & \text { Domain }\end{array}$

The knowledge issue, or the person's information need, is what drives the seek out information. The goal is to figure out why the issue exists and what function the knowledge gleaned from the quest would serve. Date and time stamps are used to contextualize the proposed solution in the event that it leads to the development of the user's or collaborators' increased awareness. The user 
specifies the likely computer systems or internet sites he seeks to use it for searching, as well as the phrases he wants to use. Each of these factors contribute to determining how much a person comprehends his major issue. A category may also be blamed for the customer's issues.

The customer is provided with a list of regions from the server to choose from and. If he cannot find a region that is important to his query, he can create a new region that will be linked to the document's database list instantly. This is used to manage "mapping" amongst knowledge challenges and consumers in identify possible partners based on the related realms. The identified problem and its explanation are saved in the base of knowledge to be used again in the future.

\subsection{Clarification Agent}

On the basis of a provided model structure, the clarification agent is in charge of all annotation and effective interactions amongst consumers. If one user requests cooperation from that other while the latter agrees, the question context between them will become a mutual question framework [25]. The owner's preliminary problem description assists the partner in comprehending the question context and, as a result, seeks to explain the problem context based on his interpretation. This method of clarification entails simultaneous correspondence and annotations on the problem context, in which the two users combine and separate their individual understandings of the problem to arrive at a common undecided

The joint feedback and consensus on data systems that can be used for searches and phrases that will best reflect the shared data need is a part of the compression process. They will now begin building and reinterpret queries to depict the knowledge issue using the information management system they have chosen. This framework allows you to switch between these points. They can gain new knowledge while formulating queries based on the retrieved data. As a consequence, they can need to change the problem's context.

\subsection{Online Navigator Agent}

The relation to the internet explorer of both the network over which the device is mounted is managed by the online navigator agent. This is done by XPCOM inter-process interaction within this device and also the network's Mozilla engine (cross platform component object model). This gives users a common system from which they can attach towards any online data system or search tool mostly during co - creation process.

\subsection{Connection agent}

The connection agent handles all demands for and acceptance of direct collaboration.

\subsection{Knowledge base exploration}

This framework also lets people to see previous user issues pertaining to their question. As want to have a client to obtain a previous overview of that he can work with it in the cooperative approach by beginning here. He could also create a basis of debt based on this. Traditionally, seeing specific users' past performance enable customer to have seen a solution to your dilemma, but some believe that reviewing previous related practices, and even the owners of those actions, will advise a user about whom to pursue direct cooperation with, help the consumer to profit from the implicit (yet unspoken) awareness of all other consumer.

In order to benefit from these past activities and other knowledge in the knowledge base, this was to allow user to exploit the knowledge base using the EQuA"te [27] search functionalities which is made up four cognitive phases:

- Exploration phase

- Query phase

- Analysis phase

- Annotation phase

Exploration phase: During this stage of development, the customers can find the body of knowledge for knowledge. The presumption is that a user with neither a complete sense of his details require or indeed a clear understanding of how and why the IRS webpage operates should really be able to view and analyze the information base's relevant data. During the discovery process, a consumer learns more about his it need as well as how the CIRS works.

The query process helps a consumer and place his newly gained knowledge (from the discovery phase) to use for the implementing a question to convey his information requirements.

The consumer will infer range of assessment for critical thinking from the got data during the analysis process. The development of added value to knowledge occurs during this process. Mining techniques can be performed in the research process by crossing or more characteristics and inferring information from the interaction among these.

By comparing the applied load only with knowledge issue variable during the analysis process, a user may find a possible associate. The person's power in the specific problem, including its authority in the users' network, are taken into account when matching. The number of significant past issues in which the user assisted in their 
settlement defines the user's status in the problem domain. The number of participants with whom a person knows interacted is used to measure the customer's influence in the system.

Annotation phase: this step enables the user to rate and report on the file that was collected. It is a stage in which new information can be created. Annotations by users are considered to create value to documents, and they're used to decide if a file is relevant to a user's information needs. In other phrases, it allows and for contextualization of results.

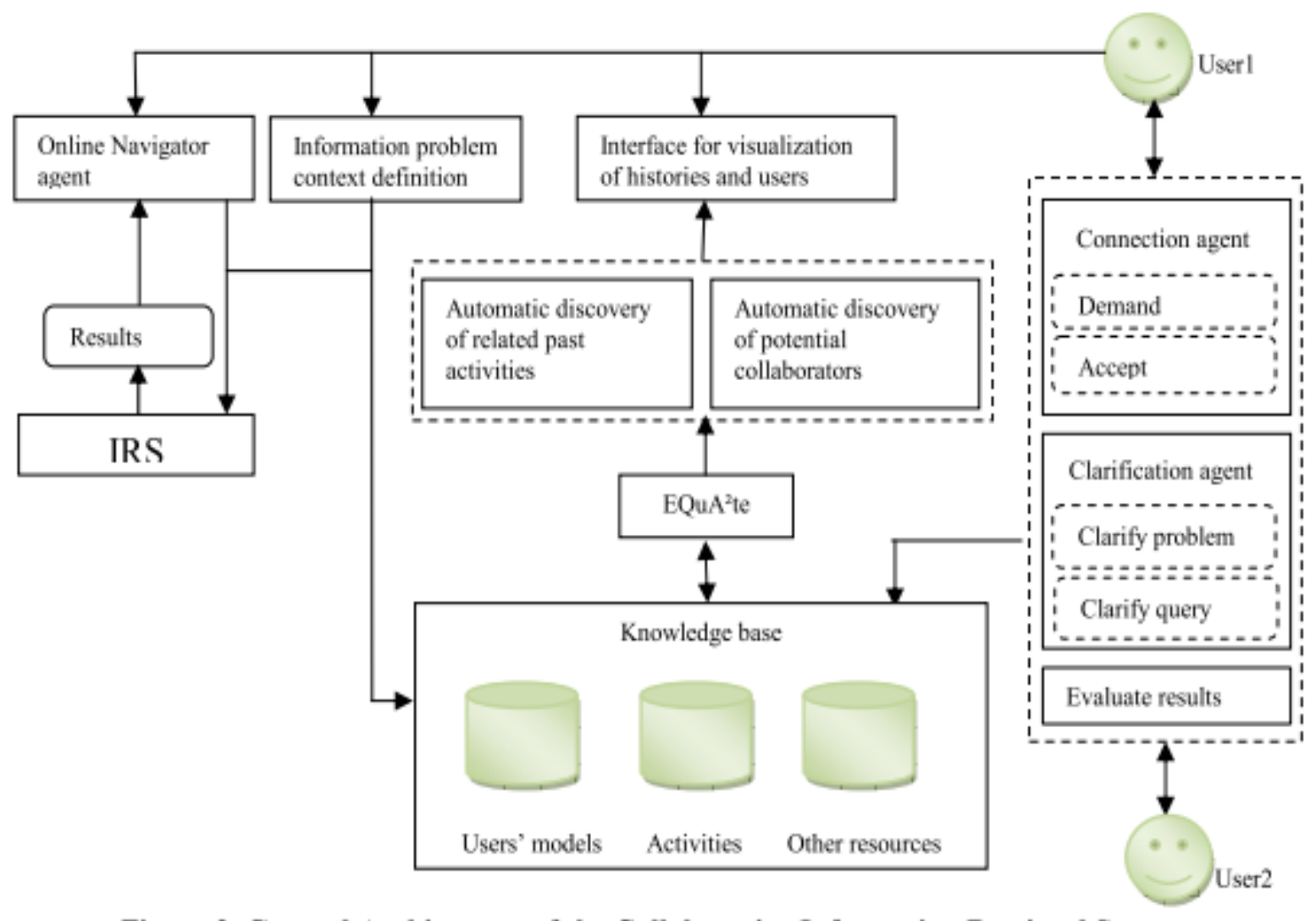

Figure 2 General Architecture of the Collaborative Information Retrieval System

\section{CONCLUSIONS AND FUTURE WORK}

In this document, as demonstrated how consumers in the IR display interactive knowledge behavior in realworld situations. As stressed that designing a framework to support this collaborative behavior ought not be restricted to an intelligence strategy, and should also involve achieving specific coordination amongst consumers to encourage tacit information sharing. Before moving on to collective report generation and result analysis, we clarified that collective information retrieval would start with a negative view and a mutual understanding of the issue. The issue description interface is the initial step in CIRS, which was created to promote information sharing in Information Retrieval. Keep looking into the CIB of consumers and how much it affects information exchange in group grids. We'll also keep working on the process to increase efficiency.

\section{REFERENCES}

[1] S. Amershi, and M.R. Morris, CoSearch: A System for Co-located Collaborative Web Search, Proceedings of CHI, Florence, Italy (2008)

[2] A David, and D. Bueno, Towards Cooperative Information Retrieval System with User Modeling, In $5^{\text {th }}$ International Conference on Information Systems Analysis and Synthesis - ISAS'99. Orlando, USA, 1999. Retrieved December 10, 2007, from http://hal.inria.fr/inria/00098974/en/

[3] A David, and O. Thiery, "Application of "EQuA2te" Architecture in Economic Intelligence," Information and Communication Technologies applied to Economic Intelligence - ICTEI'2002 Ibadan, Nigeria, pp. 32 - 38, (2002)

[4] R. Fidel, H. Bruce, AM. Pejtersen, S. Dumais, J. Grudin, and S. Poltrock, "Collaborative Information Retrieval (CIR)," The New Review of Information Behavior Research: Studies of Information Seeking in Context, 1, pp. 235-247 (2001) 
[5] J. Foster, "Collaborative Information Seeking and Retrieval," Annual Review of Information Science and Technology, 40, pp.329-356 (2006)

[6] L. Fu, D. Gob, S. Foo, and Y. Supangat, Collaborative Querying for Enhanced Information Retrieval, R. Heery and L. Lyon (Eds.): ECDL 2004, LNCS 3232, pp. 37\&388 , (2004)

[7] M. Ginsburg, "Anaotate! A tool for collaborative information retrieval," Proceedinga of Seventh IEEE International Workshop on Enabling Techoologies: Infrastmcture for Collaborative Enterprises, June 17-19, 1998, pp.75-81, (1998)

[8] D.H. Goh, L. Fu, and S. Foo, Collaborative Querying Using the Query Graph Visualize, Online Information Review, 29(3), 266-282, (2005)

[9] S.A. Golder, and B.A. Huberman, 'Usage Patterns of Collaborative Tagging Systems, Journal of Information Science, 32, 198-208, (2006)

[10] M. Karamuftuoglu, 'Collaborative Infurmation Retrieval: Toward a Social Informatics View of IR Interaction, Journal of the American Society of Information Science, vol 49(12), pp.1070-1080, (1998)

[11] J. Longchamp, Le Travall Cooperatıf Et Ses Technowgies, Lavoisier, Paris, (2003)

[12] H. Maghrebi, and A. David, Open System for Indexing and Retrieving Multimedia Information, Proceedings of $36^{\text {th }}$ Congres Anaue1 de I' Aasociation Canadienoe des Sciences de I'Information (ACSI), University of British Columbia, Vancouver, June 5 -7, (2008)

[13] V. Odumuyiwa, and A. David, A Communication Model for Knowledge Sharing in Collaborative Information Retrieval, International Conference on Knowledge Management (ICKM 2008), 23-24 October, 2008, Columbus, Ohio, USA, (2008)

[14] V. Odumuyiwa, and A. David, Collaborative Information Retrieval among Economic Intelligence Actors, Proceedings of the Fourth International Conference on Collaboration Techoologies (CollabTech 2008), Wakayama, Japan, August 2008, pp. 21-26, (2008)

[15] C. Robert, Collaborative Anaotation Creation and Access in a Multimodal Environment with Heterogeneous Devices for Decision Support and for Experience Sharing, IEEE International Conference on Sensor Networks, Ubiquitous and Trustworthy Computing, SUTC'08, 11-13 June 2008, pp.519-523, (2008)

[16] N.C. Romano, D. Roussinov, J.F. Nunamaker, and H. Chen, Collaborative Information Retrieval Environment: Integration of Information Retrieval with Group Support Systems, Proceedings of the $32^{\text {nd }}$ Hawaii Intemational Conference on System sciences. 501 , (1999)

[17] A. Sutcliffe, and M. Eunis, Towards a Cognitive Theory of Information Retrieval, Interacting with Computers, vol. 10, pp. 321-351, (1998)

[18] M.B. Twidale, D.M. Nichols, and C.D. Paice, "Browsing is a Collaborative Process," Information Processing
Management: an International Journal, 1997, 33(6), 761783.

[19] X. Zhang, and Y. Li, An Exploratory Study on Knowledge Sharing in Information Retrieval, Proceedings of the 38th Hawaii International Conference on System Sciences, (2005)

[20] C. Danilowicz, H.C. Nguyen, N.T. Nguyen, Model of User Profiles and Personalization for Web-based Information Retrieval Systems, In Abramowicz W. (Ed.), Knowledge Based Infurmation Retrieval and Filtering from Interaet, Kluwer Academic Publisher, pp.121-136, (2003)

[21] Shivanna, Prakasha \& Shashidhar, Halagappa \& Raju, Gt, Structured Intelligent Search Engine for effective information retrieval using query clustering technique and Semantic Web. 688-695. 10.1109/IC3I.2014.7019820, (2014)

[22] Kumar, Sushant \& Raju, Gt \& Shivanna, Prakasha, Improving Browsing Experience through Query Optımızation and Personalized Result Restructuring. IRAJ. 2320-2106, (2013)

[23] Shivanna, Prakasha, Performance Evaluation of Query Processing Techniques in Information Retrieval, (2013)

[24] H R Shashidhara, Raju, G T, Shivanna, Prakasha, The Role of an Information Retrieval in the Current Era of Vast Computer Science Stream. IJSCE, (2013)

[25] Shivanna Prakasha, A Survey on Varıous Architectures, Models And Methodologies for Information Retrieval, IAEME. Volume 4, Issue 1. 182-194, (2013)

[26] Y. Feng, "Real Time Retrieval Technology of Vehicle Navigation and Location Information under Internet of Things Environment," 2020 International Conference on Advance in Ambient Computing and Intelligence (ICAACI), 2020, pp. 185-188, doi: 10.1109/ICAACI50733.2020.00047.

[27] M. Rakhmatullaev and J. Atadjanov, "Information Retrieval of Text Analogs Based on the CLAD Method," 2020 International Conference on Information Science and Communications Technologies (ICISCT), 2020, pp. 1-5, doi: 10.1109/ICISCT50599.2020.9351465.

[28] N. Z. Tawfeeq, W. S. Abed and O. G. Ghazal, "A semantic model of morphological information retrieval: A comparative accumulative analysis," 2020 2nd Annual International Conference on Information and Sciences (AiCIS), 2020, pp. 1-6, doi: 10.1109/AiCIS51645.2020.00011.

[29] Bhuvaneswary, N., S. Prabu, K. Tamilselvan, and K. G. Parthiban. "Efficient Implementation of Multiply Accumulate Operation Unit Using an Interlaced Partition Multiplier." Journal of Computational and Theoretical Nanoscience 18, no. 4 (2021): 1321-1326.

[30] Bhuvaneswary, N., S. Prabu, S. Karthikeyan, R. Kathirvel, and T. Saraswathi. "Low Power Reversible Parallel and Serial Binary Adder/Subtractor." Further Advances in Internet of Things in Biomedical and Cyber Physical Systems (2021): 151 
[31] K. Yu, L. Tan, M. Aloqaily, H. Yang, and Y. Jararweh, "Blockchain-Enhanced Data Sharing with Traceable and Direct Revocation in IIoT", IEEE Transactions on Industrial Informatics, doi: 10.1109/TII.2021.3049141.

[32] K. Yu, L. Lin, M. Alazab, L. Tan, B. Gu, "Deep LearningBased Traffic Safety Solution for a Mixture of Autonomous and Manual Vehicles in a 5G-Enabled Intelligent Transportation System", IEEE Transactions on Intelligent Transportation Systems, doi: 10.1109/TITS.2020.3042504.

[33] K. Yu, M. Arifuzzaman, Z. Wen, D. Zhang and T. Sato, "A Key Management Scheme for Secure Communications of Information Centric Advanced Metering Infrastructure in Smart Grid," IEEE Transactions on Instrumentation and Measurement, vol. 64, no. 8, pp. 2072-2085, August 2015. https://ieeexplore.ieee.org/document/7138617

[34] Subramani, Prabu, Ganesh Babu Rajendran, Jewel Sengupta, Rocío Pérez de Prado, and Parameshachari Bidare Divakarachari. "A block bi-diagonalization-based pre-coding for indoor multiple-input-multiple-outputvisible light communication system." Energies 13, no. 13 (2020): 3466.

[35] Rajendrakumar, Shiny, and V. K. Parvati. "Automation of irrigation system through embedded computing technology." In Proceedings of the 3rd International Conference on Cryptography, Security and Privacy, pp. 289-293. 2019.

[36] Seyhan, Kübra, Tu N. Nguyen, Sedat Akleylek, Korhan Cengiz, and SK Hafizul Islam. "Bi-GISIS KE: Modified key exchange protocol with reusable keys for IoT security." Journal of Information Security and Applications 58 (2021): 102788.

[37] Nguyen, Tu N., Bing-Hong Liu, Nam P. Nguyen, and Jung-Te Chou. "Cyber security of smart grid: attacks and defenses." In ICC 2020-2020 IEEE International Conference on Communications (ICC), pp. 1-6. IEEE, 2020.

[38] Rajendran, Ganesh B., Uma M. Kumarasamy, Chiara Zarro, Parameshachari B. Divakarachari, and Silvia L. Ullo. "Land-use and land-cover classification using a human group-based particle swarm optimization algorithm with an LSTM Classifier on hybrid preprocessing remote-sensing images." Remote Sensing 12, no. 24 (2020): 4135.

[39] L. Zhen, A. K. Bashir, K. Yu, Y. D. Al-Otaibi, C. H. Foh, and P. Xiao, "Energy-Efficient Random Access for LEO Satellite-Assisted 6G Internet of Remote Things", IEEE Internet of Things Journal, doi: 10.1109/JIOT.2020.3030856.

[40] L. Zhen, Y. Zhang, K. Yu, N. Kumar, A. Barnawi and Y. Xie, "Early Collision Detection for Massive Random Access in Satellite-Based Internet of Things," IEEE Transactions on Vehicular Technology, vol. 70, no. 5, pp. 5184-5189, May 2021, doi: 10.1109/TVT.2021.3076015.

[41] L. Tan, K. Yu, A. K. Bashir, X. Cheng, F. Ming, L. Zhao, X. Zhou, "Towards Real-time and Efficient
Cardiovascular Monitoring for COVID-19 Patients by 5G-Enabled Wearable Medical Devices: A Deep Learning Approach", Neural Computing and Applications, 2021, https://doi.org/10.1007/s00521-021-06219-9

[42] Pham, Dung V., Giang L. Nguyen, Tu N. Nguyen, Canh V. Pham, and Anh V. Nguyen. "Multi-topic misinformation blocking with budget constraint on online social networks." IEEE Access 8 (2020): 78879-78889. 